\title{
EMOTION RECOGNITION BASED ON VARIOUS PHYSIOLOGICAL SIGNALS - A REVIEW
}

\author{
Supriya Londhe ${ }^{1}$ and Rushikesh Borse ${ }^{2}$ \\ ${ }^{1}$ Giesecke and Devrient MS India Private Limited, India \\ ${ }^{2}$ Electronics and Telecommunication Engineering, MIT Academy of Engineering, India
}

\begin{abstract}
Emotion recognition is one of the biggest challenges in human-human and human-computer interaction. There are various approaches to recognize emotions like facial expression, audio signals, body poses, and gestures etc. Physiological signals play vital role in emotion recognition as they are not controllable and are of immediate response type. In this paper, we discuss the research done on emotion recognition using skin conductance, skin temperature, electrocardiogram (ECG), electromyography (EMG), and electroencephalogram (EEG) signals. Altogether, the same methodology has been adopted for emotion recognition techniques based upon various physiological signals. After survey, it is understood that none of these methods are fully efficient standalone but the efficiency can be improved by using combination of physiological signals. The study of this paper provides an insight on the current state of research and challenges faced during emotion recognition using physiological signals, so that research can be advanced for better recognition.
\end{abstract}

Keywords:

Physiological Signals, Skin Conductance, EMG, ECG, EEG

\section{INTRODUCTION}

Emotion is a psycho-physiological state of a human being which describes a person's temperament. Although human emotional experience plays utmost important role in human lives, our scientific knowledge about human emotions has its own limitations. Hence, an ability to detect and recognize ones' emotional state is essential in the improvement of artificial intelligence part of Human Machine Interaction (HMI). Emotion recognition is widely used in medical, defense, lie detection techniques, entertainment, education etc. Various findings from neuroscience, cognitive science and psychology suggest that emotions are important in human intelligence development, social interaction, perception, learning, and so on.

Physiological signals are generated by the body during the functioning of various physiological systems. Hence, physiological signals hold information which can be extracted from these signals to find out the state of the functioning of these physiological systems. The process of extracting information can be very simple as feeling the pulse to find the state of heart beats and it can be complex which may require analysis of the structure of tissue by a sophisticated machine.

Human beings understand each other's emotion by means of text, speech and facial expressions, but there are many limitations to above said techniques, like emotion recognition using text is applied to words or sentences in a particular language so it is quite difficult to develop a universal system using text. Emotion recognition using facial expressions has many advantages but facial expressions are not always linked with inner emotions thus facial expressions can be consciously controlled. As a result, emotions cannot be recognized accurately. Emotion recognition using speech is much simpler than the above two but speech varies from person to person with different culture or geography hence speech recognition technique is also not feasible. To cope up with the limitations.

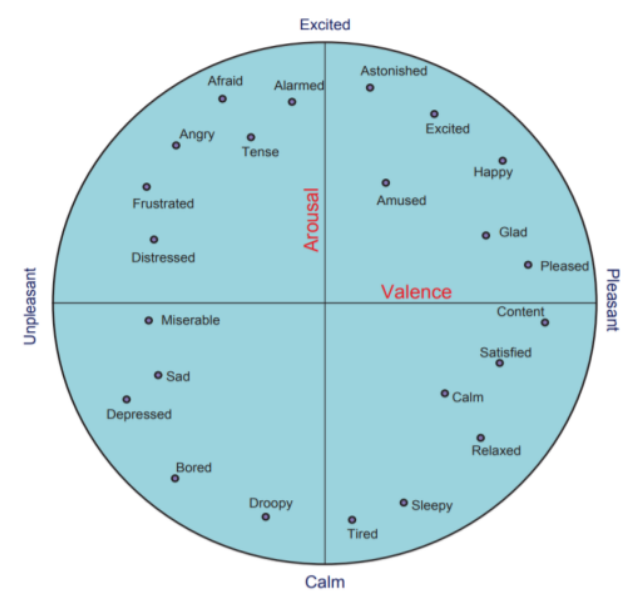

Fig.1. Arousal/Valence Space Description of Emotions [16]

For the above said methods, physiological signals can be used. Every human being generates various signals from different parts of the body. These signals are termed as physiological signals required for analysis of emotions using HCI [16]. There are many advantages of monitoring physiological patterns of the body controlled by the nervous system which is getting impacted by human emotions. There are various sensors which can be used for collecting physiological signals such as ECG, EMG, EEG, skin conductance, skin temperature and Blood Volume Pulse (BVP) [24].

The discrete emotional model claims the presence of some basic and universally standard emotions irrespective of culture or geography [16]. Several psychologists have suggested different categories of emotions. But there has been a considerable agreement in the following six emotions happiness, sadness, surprise, anger, disgust, and fear. The dimensional model categorizes emotions based on the scales and can be characterized on valance and arousal (Fig.1). Valance represents an emotional state, unpleasant to pleasant which varies from negative to positive. Arousal indicates the journey of human emotions calm to excite and travels from low to high.

In this paper, we present a review of the recent advancements in emotion recognition techniques and corresponding research using physiological signals; in specific to the emotion elicitation stimuli, feature extraction and classification methodologies. 
The remaining part of this paper is organized as follows: section 2 gives an overview of the methodology for emotion recognition. Section 3 describes emotion recognition based on skin conductance and EMG. Section 4 talks about emotion recognition based on ECG. Section 5 presents an overview of previous research works based upon EEG emotion recognition technique. Section 6 concludes with the findings of this review on emotion recognition.

\section{METHODOLOGY FOR EMOTION RECOGNITION}

The implementation of the study of emotion recognition using physiological signals follows various steps [24] such as data acquisition, pre-processing, feature extraction, feature selection, classification and finally recognizing emotion. This can be seen in Fig.2. The implementation method remains more or less the same for different physiological signals.

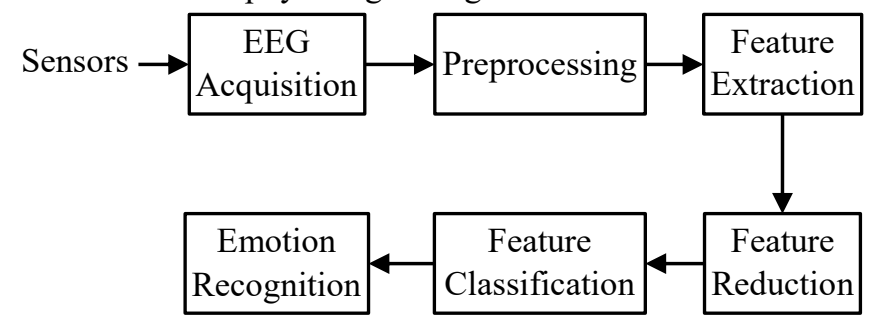

Fig.2. General Block diagram of emotion recognition system [24]

Data acquisition is the process of collecting data in terms of physiological signals through various sensors. Collected raw physiological signals contain a lot of artifacts and noise which needs to be removed. This can be done by pre-processing of signals. After removing noise, various salient features of the signal need to be identified. Emotion recognition is to be done using feature extraction technique. There are various features which could be taken into consideration but one needs to consider the features giving out efficient results by making use of feature selection. The results from feature extraction are classified in various classes, where each class corresponds to a particular emotion. This is done with the help of different classifiers which comes under classification process. The step by step description of the said block diagram (Fig.2) is given in below sections.

\subsection{DATA ACQUISITION}

This is the first step where the emotions are induced by exposing the person to particular stimuli. Different stimuli can be used such as sounds, images and videos to gathering high quality database [22]. Various emotion elicitation methods such as International Affective Picture System (IAPS) database [17] [26] [28], International Affective Digitized Sounds (IADSs), music video clips [15], musical clips [31] are used by researchers to elicit the target emotions. To record the physiological signals, different types of sensors are used. To measure Skin Conductivity Electrodermal Activity (EDA) is used [24] [2]. Electrodermal activity (EDA) can easily be measured from the body surface and it represents the activity of the autonomic nervous system. It is also called as galvanic skin response [32]. Variations in the skin temperature (SKT) mainly come from localized changes in blood flow caused by vascular resistance or arterial blood pressure [24].
Electromyography (EMG) is recorded by using a Quadripolar electrode placed on the muscle [24] [12]. EMG is a technique used to record electrical potential created by muscle membranes. Electroencephalogram (EEG) is measured using $\mathrm{Ag} / \mathrm{AgCl}$ electrodes cap and BIOPAC electrode lead set (SS2L) [22]. EEG signals refer to the activity of the Central Nervous System (CNS). EPIC sensor is used to measure the ECG signals. ECG device detects the electrical changes on the skin of the heart muscle during each heartbeat [24] [19]. These various recorded physiological changes are undertaken for preprocessing.

\subsection{PREPROCESSING}

Preprocessing stage mainly comprises of removal of noise, artifacts, and other external interferences. Artifacts and interference in raw physiological signals are removed by using adaptive filter, Laplacian filter, visual inspection and Independent component analysis (ICA). Low pass IIR filter, Butterworth lowpass filter, elliptic filter, moving average filter are also used for removing noise [4] [8]. To avoid power line contamination notch filter is used. To avoid power line contamination $60 \mathrm{~Hz}$ notch filter is sometime used. The pattern of EEG signal spreads over the frequency range of 1-50 Hz. The essential data is extracted by using $0.2-45 \mathrm{~Hz}$ band pass filter. Heartbeat generates the artifacts in EMG signal. These artifacts are removed by using adaptive band pass filter.

\subsection{FEATURE EXTRACTION}

After the preprocessing, different feature extraction methods are applied on the preprocessed signal. The main task of feature extraction is to derive the salient features which can map the physiological data into consequent emotion states. Feature extraction method are divided into three domains: Time Domain, Frequency Domain and Time-Frequency Domain [18] [34]. Some feature extraction methods are common for all physiological signals which are as follows:

\subsubsection{Statistical Methods:}

From each signal, six statistical features are computed [31] [26] [15]. Let $X(n)$ represent the value of the $n^{\text {th }}$ sample of raw signals, where $n=1,2, \ldots, n$. Let $\bar{X}(n)$ refer to the normalized signal:

$$
\bar{X}(n)=\frac{X(n)-\mu_{x}}{\sigma_{x}}
$$

The basic six statistical features are:

The mean of the raw signal.

$$
\mu_{x}=\frac{1}{N} \sum_{n=1}^{N} X(n)
$$

The standard deviation of the raw signal.

$$
\sigma_{x}=\sqrt{\frac{1}{N} \sum_{n=1}^{N}\left(X(n)-\mu_{x}\right)^{2}}
$$

The mean of the absolute values of the first differences of the raw signal.

$$
\delta_{X}=\frac{1}{N-1} \sum_{n=1}^{N-1}|X(n+1)-X(n)|
$$

The mean of the absolute values of the first differences of the standardized signal. 


$$
\bar{\delta}_{X}=\frac{\delta_{X}}{\sigma_{x}}
$$

The mean of the absolute values of the second differences of the raw signal.

$$
\gamma_{X}=\frac{1}{N-2} \sum_{n=1}^{N-2}|X(n+2)-X(n)|
$$

The mean of the absolute values of the second differences of the standardized signal.

$$
\bar{\gamma}_{X}=\frac{\gamma_{X}}{\sigma_{x}}
$$

Euclidean Distance: Let $X_{n e u}$ represent the signal to the neutral emotion [28]. The Euclidean distance between the signal to such an emotion and the signal $X_{\text {neu }}$ is calculated as follows:

$$
D_{X}=\sqrt{\frac{1}{N-1} \sum_{n=1}^{N}\left|X(n)-X_{n e u}\right|^{2}}
$$

Spectral Coherence Function: This feature represents the correspondence of two signals $X_{i}$ and $X_{j}$ at each frequency [28]. It is defined as:

$$
C_{i j}(f)=\frac{\left|X_{i j}(f)\right|^{2}}{X_{i i}(f) X_{i j}(f)}
$$

where, $C_{i j}(f)$ is the cross-spectral density between $i$ and $j$, and $X_{i i}(f)$ and $X_{j j}(f)$ are the spectral density of $i$ and $j$ respectively.

\subsection{FEATURE REDUCTION}

There is large amount of possible features that are extracted from the various bio-signals. This problem of high dimensional feature space is solved by feature selection. Feature reduction could help to increase the speed, performance and stability of the computation [18] [34]. In order to select the relevant features for efficient emotion classification, various feature reduction algorithms such as Genetic Algorithm (GA) [26] [20], MinRedundancy-Max-Relevance (MRMR) [15] [36], ReliefF and Effect-Size (ES)-Based Feature Selection etc., has been used by various researchers. GA-based feature selection method is quite reliable and powerful which results in finding the best feature subset for this particular application. The MRMR method enhances the Min-Redundancy means to minimize the average mutual information between two features and MaxRelevance means to maximize average mutual information between each feature and the target vector simultaneously [18]. In the releifF method [18], feature selection is done by estimating a quality weight for each feature. Sequential forward selection of features is carried out in Effect-Size (ES) Based Feature Selection [18].

\subsection{CLASSIFICATION}

The extracted features are classified for different kinds of emotions like Happy, sad and Neutral etc. There are so many methods available to classifying the features from the physiological signal. The classification can be performed by using Support Vector machine (SVM) [5] [33] [1], k-Neural Networks (k-NN) [7] [36], Quadratic Discriminant Analysis (QDA), Artificial Neural Network (ANN), Linear Discriminant Analysis (LDA) [20] [27], Fisher linear discriminant [7], Mahalanobis Distance (MD) [23] and Multilayer Perceptron (MLP). Different kernel functions are available for SVM classifier. They are linear kernel, polynomial kernel, RBF kernel and Sigmod kernel function [11]. It has been observed that the performance of SVM classifier is better than LDA classifier [37].

\section{EMOTION RECOGNITION BASED ON SKIN CONDUCTANCE AND EMG}

\subsection{SKIN CONDUCTANCE}

Human body has continuous variation in the electrical characteristics of the skin. This property of the human body is known as electrodermal activity (EDA) [24]. Theory of EDA holds that skin resistance varies with the state of sweat glands in the skin. Sweating is controlled by the sympathetic nervous system [3] and skin conductance is an indication of psychological or physiological arousal. If the sympathetic branch of the autonomic nervous system is highly aroused, then sweat gland activity also increases, which in turn increases skin conductance.

Wu et al. [9] proposed a method to recognize six emotions (surprise, anger, fear, disgust, grief, and happiness) from GSR signals. For emotion recognition, 30 different feature extraction methods are used. To improve recognition rate Immune Hybrid Particle Swarm Optimization (IH-PSO) was used to select the feature subsets of different emotions. Yamakoshi et al. [11] developed a new method for driver stress detection from differential skin temperature measurement. For this 25 subjects were investigated under environment-controlled conditions and acquired physiological signals, including facial skin temperature.

\subsubsection{Limitations:}

This signal changes if the skin is sweaty. It is related to stress situations and another affects.

\subsection{EMG}

Electromyography (EMG) is the recording and analysis of the bioelectric activity of skeletal muscles. Electromyography is measuring the electrical signal associated with muscle activation. This may be voluntary or involuntary muscle contraction [24]. EMG can be recorded by two types of electrodes: invasive electrode (so-called wire or needle electrodes) and non-invasive electrode (so-called surface electrode). Wire or needle electrodes record action potentials of individual muscle fiber [12].

Gouizet et al. [6] developed a new method for detecting negative emotion recognition by using EMG signals. Negative emotions of interest in this work are: fear, disgust and sadness. Different feature extraction method such as signal Rectification, Quadratic mean, Average Energy, Signal Integration, Wavelet transform are used. For classification, Gaussian kernel of SVM technique provides good recognition rates. Zhu [37] presented a new emotion recognition using the BP neural network. It is a multi-level error feedback network. BP neural network was employed for solving linear equations used to achieve the classification makes the training time greatly reduced and the recognition rate can reach more than $83.33 \%$. In [35], the author proposed emotional pattern recognition using Least Squares Support Vector Machine (LSSVM). For this, wavelet transform is used which has multi scale decomposition level. By using this method, the recognition rate reached up to $91.67 \%$. 


\subsubsection{Limitations:}

Facial electromyogram can be very useful for emotion recognition but the electrodes fixed on face are too uncomfortable and intrusive for a user and then useless in practice

\section{EMOTION RECOGNITION BASED ON ECG}

The electrocardiogram ECG is a tool that is used to assess the electrical and muscular functions of the heart [7], [24]. Heart is a two stage electrical pump. Heart's electrical activity can be measured by electrodes placed on the skin. Standardized electrode placement system has been developed for routine ECG. Ten electrodes are required to produce 12 electrical views of the heart. An electrode lead, or patch, is placed on each arm and leg and six electrodes are placed across the chest wall. The signals received from each electrode are recorded [10]. The printed view of these recordings is the electrocardiogram. By comparison, a heart monitor requires only three electrode leads one each on the right arm, left arm, and left chest. It only measures the rate and rhythm of the heartbeat.

However, conventional electrodes possess various disadvantages. There are not conducive for long-term use. For nonclinical settings they are quite expensive and hence capacitive (insulated) electrodes are used which can register ECG [19] signals without conductive contact to the body - even through clothes such electrodes are called EPIC [8] (Electric Potential Integrated Circuit). The signals produced using the electrodes has some features which needs to extracted, some of those features are:

\subsection{FISSION (HHT)}

In fission process, two features are extracted: instantaneous frequency \& amplitude and these are obtained by applying Hilbert transform on each IMF [29].

$$
H\left[c_{i}(t)\right]=\frac{1}{\pi} P V \int_{-\infty}^{\infty} \frac{c_{i}\left(t^{\prime}\right)}{t-t^{\prime}} d t^{\prime}
$$

where $P V$ indicates the Cauchy principal value. One can define the following analytical signals

$$
Z_{i}(t)=c_{i}(t)+j H\left[c_{i}(t)\right]=\alpha_{i}(t) e^{j \theta} i(t)
$$

For IMFs, the instantaneous frequency $f_{i}(t)$ can be computed as:

$$
f_{i}(t)=\frac{1}{2 \pi} \frac{d \theta_{i}(t)}{d t}
$$

\subsection{FUSION (HHT)}

Fusion process, aims information merging of each IMF to compute the mean frequency [29]. To find mean frequency, one can use weighted mean instantaneous frequency (WMIF) of each IMFs $c_{i}(t)$ with $N$ samples. It is defined as,

$$
W M I F(i)=\frac{\sum_{j=1}^{N} f_{i}(j) A_{i}^{2}(j)}{\sum_{j=1}^{N} A_{i}^{2}(j)}
$$

The mean frequency (MNF) [5] is defined from WMIF is as:

$$
M N F=\frac{\sum_{i=1}^{n}\left\|a_{i}\right\| W M I F(i)}{\sum_{i=1}^{n}\left\|A_{i}\right\|}
$$

Along with mean frequency, statistical features (min, max, standard deviation, range and median) are also extracted to generate feature vector. After extraction of the features, it needs classification therefore people use Support Vector Machine (SVM) classifier [5]. The Table.1 shows the details of the work that has been done so far in classifying the various emotions using ECG signals. Authors in [7] recorded signal for 391 subjects of age group (19-25 years old) for 4.15 minutes for classifying joy and sadness. Then 150 of ECG signal were chose for research, out of which 80 were joy emotion and 70 were sad emotion, each sample had 79 features. Each ECG signal had 50000 sampling points. The total recognition rate achieved was $84.45 \%$. Experiment and simulation results showed that it is feasible and efficient to use PSO and KNN [13] to recognize emotion states by physiological signals. Another method of feature selection is described in [34] which is based on Ant Colony System (ACS), using K-nearest

\begin{tabular}{|c|c|c|c|c|}
\hline $\begin{array}{l}\text { No. of } \\
\text { Subjects }\end{array}$ & Emotions & $\begin{array}{c}\text { Feature } \\
\text { Extraction }\end{array}$ & Classification & Accuracy \\
\hline 391 & Joy and Sad & DWT [7] & $\begin{array}{c}\text { Particle Swarm } \\
\text { Optimization } \\
\text { (PSO) and } \\
\text { KNN } \\
\end{array}$ & $84.45 \%$ \\
\hline 391 & Joy and Sad & DWT [19] & KNN & $85.14 \%$ \\
\hline 24 & Pleasure & DWT [14] & $\begin{array}{c}\text { SFFS and } \\
\text { Fisher Method }\end{array}$ & $70 \%$ \\
\hline 0 & $\begin{array}{c}\text { Happy, } \\
\text { Anger, Sad } \\
\text { and neutral }\end{array}$ & $\begin{array}{c}\text { Analysis of } \\
\text { Variance } \\
\text { (ANOVA), } \\
\text { Sequence of } \\
\text { Forward } \\
\text { Selection } \\
\text { (SFS) and } \\
\text { Sequence to } \\
\text { the } \\
\text { Selection } \\
\text { (SBS) [8] } \\
\end{array}$ & kNN and MLP & $87.5 \%$ \\
\hline 44 & $\begin{array}{l}\text { Erotica, } \\
\text { Disgust, } \\
\text { Excitement, } \\
\text { Fear, Gore }\end{array}$ & \begin{tabular}{|c|} 
Hilbert \\
Huang \\
Transform \\
(HHT) $[10]$
\end{tabular} & SVM & $76.19 \%$ \\
\hline 26 & $\begin{array}{c}\text { Negative } \\
\text { and Positive }\end{array}$ & $\begin{array}{c}\text { Wavelet } \\
\text { Transform } \\
\text { [33] }\end{array}$ & SVM & 12.4298 \\
\hline 0 & $\begin{array}{l}\text { Joy, Anger, } \\
\text { Sad and } \\
\text { Pleasure }\end{array}$ & $\begin{array}{l}\text { Hilbert- } \\
\text { Huang } \\
\text { Transform } \\
\text { (HHT) [5] }\end{array}$ & SVM & IMF: 65 \\
\hline
\end{tabular}
neighbor [14] for emotion classification, was introduced to obtain higher recognition rate and effective feature subset.

Table.1. Review of Previous Works on Emotion Recognition Using ECG Signals 
Agrafioti in [10] demonstrates the feasibility of using ECG for emotion detection. He studied Arousal detection performance for 42 subjects. Experimental setups were presented for the elicitation of active arousal and passive arousal/valence. The features used for classification are based on the instantaneous frequency (Hilbert-Huang transform) [3] and the local oscillation within every mode. Classification was performed with linear discriminants. For the three class problem (low, medium, and high arousal), the average classification rate over all subjects was $35.36 \%$. For the two class problem (low and high arousal), the average rate over all subjects was $76.19 \%$. In addition, this work differentiates between active and passive arousal.

In [38], to recognize emotions, authors have adapted the local binary pattern (LBP) and local ternary pattern (LTP) which are favorable local pattern description methods for emotion recognition. The results indicate that they effectively extracted ECG features with high accuracy. In [39], result showed that
Quranic recitation demonstrated a positive transformation of the subjects' emotions: from negative precursor emotions to calmness and happiness conditions denoted by a positive valence for the EEG and ECG signals.

In [33], the authors discussed about an improved ECG denoising method based on wavelet transform to extract the RR interval and obtain HRV. The authors, analyzed and extracted a wide range of physiological features from various domains including time, frequency and nonlinear analysis to come up with some conclusions which are: Under negative emotions, the heart rate were significantly faster, LF/HF significantly increased, while the fractal dimension decreased, indicating HRV reduced and the sympathetic nerve activity increased while vagus nerve activity decreased.

\subsubsection{Limitations:}

The disadvantage is that using electrodes is sensitive to motion of user's body what matters when one tries to recognize emotions.

Table.2. Review of Previous Works on Emotion Recognition using EEG Signals

\begin{tabular}{|c|c|c|c|c|c|c|}
\hline $\begin{array}{c}\text { No. of } \\
\text { Subjects }\end{array}$ & Electrodes & Emotions & Feature extraction & Feature Selection & Classifier & Accuracy \\
\hline 5 & 64 & $\begin{array}{l}\text { Positive, } \\
\text { negatively } \\
\text { and calm }\end{array}$ & $\begin{array}{l}\text { Statistical } \\
\text { Features [20] }\end{array}$ & $\begin{array}{c}\text { Genetic } \\
\text { Algorithm (GA) }\end{array}$ & $\begin{array}{l}\text { kNN } \\
\text { LDA }\end{array}$ & $\begin{array}{l}67 \% \\
50 \%\end{array}$ \\
\hline 16 & 3 & $\begin{array}{c}\text { Happy, } \\
\text { surprise, } \\
\text { anger, fear, } \\
\text { disgust and sad } \\
\end{array}$ & $\begin{array}{c}\text { HOC, statistical, } \\
\text { Wavelet transform [23] }\end{array}$ & - & $\begin{array}{l}\text { QDA, k-NN, } \\
\text { MD and SVM }\end{array}$ & $\begin{array}{c}62.3 \%(\text { Single ch }) \\
83.33 \%(\text { Combine } \mathrm{Ch})\end{array}$ \\
\hline 5 & 64 & $\begin{array}{l}\text { Joy, relax, } \\
\text { sad and fear }\end{array}$ & $\begin{array}{l}\text { statistical features, } \\
\text { power spectrum [15] }\end{array}$ & $\begin{array}{c}\text { minimal-redundancy- } \\
\text { maximal-relevance, } \\
\text { (MRMR) }\end{array}$ & $\begin{array}{l}\text { kNN, MLPs, } \\
\text { and SVM }\end{array}$ & $66.51 \%$ \\
\hline 6 & 62 & $\begin{array}{l}\text { Positive, } \\
\text { negative }\end{array}$ & $\begin{array}{c}\text { Diff. entropy (DE) } \\
\text { Diff. Asym. (DASM) } \\
\text { rational asym.(RASM) } \\
\text { Energy spectrum (ES) [36] }\end{array}$ & MRMR & $\begin{array}{l}\text { SVM } \\
\text { kNN }\end{array}$ & $76.56 \%-84.22 \%$ \\
\hline- & $\begin{array}{l}\text { BIOPAC } \\
\text { electrode } \\
\text { lead set }\end{array}$ & $\begin{array}{l}\text { Relax, mental } \\
\text { task, memory } \\
\text { related task, } \\
\text { pleasant and } \\
\text { fear }\end{array}$ & $\begin{array}{l}\text { statistical features, } \\
\text { FFT, DWT [1] }\end{array}$ & - & SVM & $52.14 \%$. \\
\hline 57 & 8 & $\begin{array}{l}\text { Valance, } \\
\text { Arousal }\end{array}$ & DWT and HOC [32] & $\begin{array}{l}\text { nonnegative sparse } \\
\text { PCA (NSPCA) }\end{array}$ & $\begin{array}{c}\text { Feed-forward } \\
\text { neural network }\end{array}$ & $\begin{array}{l}67.39 .4 \% \text { (Valance) } \\
65.859 .7 \% \text { (Arousal) }\end{array}$ \\
\hline 6 & 62 & $\begin{array}{c}\text { Positive, } \\
\text { neutral } \\
\text { and Negative }\end{array}$ & Wavelet transform [30] & - & $\begin{array}{c}\text { SVM linear, } \\
\text { RBF } \\
\text { polynomial } \\
\text { Sigmod kernel }\end{array}$ & $62.16 \%-79.86 \%$ \\
\hline 60 & 64 & $\begin{array}{l}\text { happy, curious, } \\
\text { angry, sad, } \\
\text { quiet }\end{array}$ & $\begin{array}{l}\text { ERP, statistical, HOC, } \\
\text { hjorth, NSI, FD, bandpower, } \\
\text { HOS, DWT, MSCE, HHS, } \\
\text { diff. and rational asym. [18] }\end{array}$ & $\begin{array}{c}\text { mRMR } \\
\text { ReliefF } \\
\text { Effect-Size (ES) }\end{array}$ & QDA & $35.9 \%$ \\
\hline 32 & 4 & $\begin{array}{c}\text { happy, sad, } \\
\text { anger, } \\
\text { disgust, neutral, }\end{array}$ & PSD, Energy [27] & - & $\begin{array}{l}\text { SVM } \\
\text { LDA }\end{array}$ & $\begin{array}{l}74.13 \% \\
66.50 \%\end{array}$ \\
\hline
\end{tabular}




\section{EMOTION RECOGNITION BASED ON EEG}

The human brain is one of the complex and main organ of human body. It is made up of more than 100 billion nerves. The brain is responsible for all kinds of body's action and reaction. Electroencephalography (EEG) technique is used to measure the electrical activity of the neurons inside the brain. When the neurons are active, they produce an electrical potential. This electrical potential is recorded through the electrodes, which are placed on the scalp of the brain [2]. The electrical potential is nothing but the voltage between pairs of these electrodes which are then filtered, amplified, and recorded. Brain waves have been classified into four different frequency bands [21]: delta $(0-4 \mathrm{~Hz})$, theta $(4-8 \mathrm{~Hz})$, alpha $(812 \mathrm{~Hz})$ and beta $(12-30 \mathrm{~Hz})$.

In Table.2, there is a short review of previous work in emotion recognition field using EEG signals. Authors in [22] have done EEG signals recording for 32 participants aged between 19 and 37. Each participant watched 40 one-minute long excerpts of music videos. Participants rated each video in terms of the levels of arousal, valence, like/dislike, dominance and familiarity. Petrantonakis et al. [23] proposed higher order crossing (HOC) method for emotion recognition. The accuracy for single channel is $62.3 \%$ using QDA classifier and for combination channel is $83.33 \%$ using SVM classifier.

Authors in [40], have detected human emotion with a singlechannel commercial BCI device and were tested on human test subjects using a deep learning neural network and accuracy above $87 \%$ was achieved.

Jenke et al. [18] studied various methods of feature extraction and feature selection for emotion recognition from EEG. Features are distinguished in time domain, frequency domain and timefrequency domain. Some features are calculated from the recorded signal of a single electrode and some features required combination of electrode. There is different type of feature selection methods based on selection of feature type and selection of electrode locations. Some of these features are:

\subsection{HIGHER ORDER CROSSING (HOC)}

This feature is based on oscillation about zero level. Here a sequence of high-pass filters is applied to the time series $Z(t)$ and count the number of zero-crossings in the filtered time series [23]. HOC estimate by,

$$
D_{k}=N R Z\left\{\nabla^{k-1} Z(t)\right\} .
$$

\subsection{HIGHER ORDER SPECTRA (HOS)}

HOS is a frequency domain feature. HOS is based on bispectra and bicoherence [18]. In HOSA toolbox Bis and Bic function are available for bispectra and bicoherence respectively. The Bis represents the Fourier Transform of the third order moment of the signal:

$$
\operatorname{Bis}\left(f_{1}, f_{2}\right)=E\left[\xi\left(f_{1}\right) \cdot \xi\left(f_{2}\right) \cdot \xi^{*}\left(f_{1}+f_{2}\right)\right]
$$

where, $E[]$ is the expectation operation and $\xi(f)$ denotes the Fourier Transform of the signal. Bic is simply the normalized bispectrum:

$$
\operatorname{Bic}\left(f_{1}, f_{2}\right)=\frac{\operatorname{Bis}\left(f_{1}, f_{2}\right)}{\sqrt{P\left(f_{1}\right) \cdot P\left(f_{2}\right) \cdot P\left(f_{1}+f_{2}\right)}}
$$

where $P(f)$ is the power spectrum. From each electrode and combinations of frequency band, different features are extracted.

In [30], the author proposed emotion recognition using wavelet transform. Different band frequencies are obtained from wavelet transform. In this system, the kernel based SVM classifier is used. The accuracy for linear kernel, polynomial kernel, RBF kernel, and Sigmod kernel is $79.86 \%, 62.16 \%, 67.57 \%$, and $62.16 \%$ respectively.

Duan et al. [36] introduced new feature named differential entropy (DE). DE performs better than the energy spectrum (ES). Combination of the classifiers increase the accuracy and stability. Techniques in [1] [15] [20] have extracted features such as statistical, Fast Fourier Transform (FFT) and Discrete Wavelet Transform (DWT) for emotion recognition. In this, the highest accuracy obtained was by DWT features $60.15 \%$ as compare to statistical features and FFT features.

Bhardwaj et al. [27] classified EEG signal into seven different emotions (happy, sad, disgust, neutral, fear, surprised, anger) by using Support Vector Machine (SVM)) and Linear Discriminant Analysis (LDA). SVM achieved a maximum accuracy of $74.13 \%$ than LDA. Khosrowabadi et al. [32] proposed to classify emotion using biologically inspired six-layer feed forward neural network. This approach obtains the accuracy of $67.3 \%$ and $65.85 \%$ for classifying valance and arousal respectively.

If an unexpected event occurs all of a sudden in case of a human being or a subject under observation/study, emotion does play an important role in decision making. At this point in time respective person or subject goes into stressful condition. Cheemalapati et al. developed algorithms for detecting stressful condition in real time [25].

\section{CONCLUSIONS}

In this paper, we presented a review of various advancements in emotion recognition research using physiological signals; in specific to the emotion elicitation stimuli, feature extraction and classification methodologies. Different physiological signals possess means advantages over each other.

In our research, we got to know that EEG has an upper hand over the other physiological signal. The methodology used for emotion recognition for the various physical signals is the same, only the features used are different. For classification, SVM is used as it is one the most complex classifier available for classifying even minute change in the values of the features. In the end, we would like to comment on the upper edge credibility of the EEG over the other physiological signals for emotion recognition.

\section{FUTURE SCOPE}

It has been understood from the above work that, EEG signal takes an upper hand over many other physiological signals. Based on the EEG signals, in recent times, people are also working on emotion recognition for COMA patients. Other application can be for stressed persons. For this, one can perform yogic practices. 
The EEG of this person can be taken before and after Yoga. Thus once can prove the efficiency of YOGA practices by showing the emotion has improved from left quadrant to right quadrant. As part from this, we would also suggest researchers to go for a multimodal approach for emotion recognition as that would give the highest accuracy. This aspect of multimodal approach has been explored in depth.

\section{REFERENCES}

[1] Tazrin Ahmed, Monira Islam and Mohiuddin Ahmad, "Human Emotion Modeling based on Salient Global Features of EEG Signal", Proceedings of IEEE International Conference on Advances in Electrical Engineering, pp. 246251, 2013.

[2] Ali S. Almejrad, "Human Emotions Detection using Brain Wave Signals: A Challenging", European Journal of Scientific Research, Vol. 44, No. 4, pp. 640-659, 2010.

[3] Liqin Fu, Xia Mao and Lijiang Chen, "Relative Speech Emotion Recognition based Artificial Neural Network", Proceedings of IEEE Pacific-Asia Workshop on Computational Intelligence and Industrial Application, pp. 140-144, 2008.

[4] Xiaowei Niu, Liwan Chen and Qiang Chen, "Research on Genetic Algorithm based on Emotion Recognition using Physiological Signals", Proceedings of IEEE International Conference on Robot and Human Interactive Communication, pp. 614-618, 2011.

[5] A.N. Paithane, D.S. Bormane and Sneha Dinde, "Electrocardiogram Signal Analysis using Empirical Mode Decomposition and Hilbert Spectrum", Proceedings of IEEE International Conference on Pervasive Computing, pp. 194-197, 2015.

[6] Gouizi, K. Maaoui and C. Reguig, "Negative Emotion Detection using EMG Signal", Proceedings of IEEE International Conference on Control, Decision and Information Technologies, pp. 690-695, 2014.

[7] Chen Defu, Cai Jing and Liu Guangyuan, "Towards Recognizing Two Emotion States from ECG Signals", Proceedings of IEEE International Conference on Computational Intelligence and Natural Computing, pp. 210-213, 2009.

[8] Xianhai Guo, "Research on Emotion Recognition based on Physiological Signal and AuBT", Proceedings of IEEE International Conference on Consumer Electronics, Communications and Networks, pp. 614-617, 2011.

[9] Guanghua Wu, Guangyuan Liu and Min Hao, "The Analysis of Emotion Recognition from GSR Based on PSO", Proceedings of International Symposium on Intelligence Information Processing and Trusted Computing, pp. 360363, 2010.

[10] Foteini Agrafioti, Dimitrios Hatzinakos and Adam K. Anderson, "ECG Pattern Analysis for Emotion Detection", IEEE Transactions on Affective Computing, Vol. 3, No. 1, pp. 102-115, 2012.

[11] T. Yamakoshi et al., "Feasibility Study on Driver's Stress Detection from Differential Skin Temperature Measurement", Proceedings of IEEE International Conference on Engineering in Medicine and Biology Society, pp. 1076-1079, 2008.
[12] Teena George, K. Shalu George and K.S. Sivanandan, "Sensing, Processing and Application of EMG signals for HAL (Hybrid Assistive Limb)", Proceedings of IEEE International Conference on Sustainable Energy and Intelligent Systems, pp. 20-22, 2011.

[13] Yu-Hao Wu, Shu-Jing Lin and Don-Lin Yang, "A Mobile Emotion Recognition System based on Speech Signals and Facial Images", Proceedings of International Conference on Computer Science and Engineering, pp. 212-217, 2013.

[14] Zhengji Long, Guangyuan Liu and C. Xuewu Dai, "Extracting Emotional Features from ECG by using Wavelet Transform", Proceedings of IEEE International Conference on Biomedical Engineering and Computer Science, pp. 1-4, 2010.

[15] Xiao-Wei Wang, Dan Nie and Bao-Liang Lu, "EEG-based Emotion Recognition using Frequency Domain Features and Support Vector Machines", Proceedings of International Conference on Neural Information Processing, pp. 734-743, 2011.

[16] Cristian A. Torres-Valencia, Hernan F. Garca-Arias, Mauricio A. Alvarez Lopez and Alvaro A. OrozcoGutierrez, "Comparative Analysis of Physiological Signals and Electroencephalogram (EEG) for Multimodal Emotion Recognition using Generative Models", Proceedings of International Conference on Image, Signal Processing and Artificial Vision, pp. 1-5, 2014.

[17] C. Maaoui and A. Pruski, "A Comparative Study of SVM Kernel Applied to Emotion Recognition from Physiological Signals", Proceedings of IEEE International MultiConference on Systems, Signals and Device, pp. 1-6, 2008.

[18] R. Jenke, "Feature Extraction and Selection for Emotion Recognition from EEG", IEEE Transactions on Affective Computing, Vol. 5, No. 3, pp. 327-339, 2014.

[19] C.A.I. Jing, L.I.U. Guangyuan and H.A.O. Min, "The Research on Emotion Recognition from ECG Signal", Proceedings of IEEE International Conference on Information Technology and Computer Science, pp. $497-$ 500,2009

[20] Z. Khalili and M.H. Moradi, "Emotion Detection using Brain and Peripheral Signals", Proceedings of IEEE International Conference on Biomedical Engineering, pp. 14, 2008.

[21] Ivan Manuel and Benito Nunez, "EEG Artifact Detection", Master Thesis, Department of Cybernetics, Czech Technical University of Prague, 2010.

[22] Sander Koelstra, Christian Muhl, Mohammad Soleymani, Jong-Seok Lee, Ashkan Yazdani, Touradj Ebrahimi, Thierry Pun, Anton Nijholt and Ioannis Patras, "DEAP: A Database for Emotion Analysis using Physiological Signals", IEEE Transactions on Affective Computing, Vol. 3, No. 2, pp. 1831, 2012.

[23] Panagiotis C. Petrantonakis and Leontios J. Hadjileontiadis, "Emotion Recognition from EEG using Higher Order Crossings", IEEE Transactions on Information Technology in Biomedicine, Vol. 14, No. 2, pp. 1-8, 2010.

[24] Revathipriya Muthusamy, "Emotion Recognition from Physiological Signals using Bio-Sensors", Technical Report, Department of Computer Science, University of Fribourg, 2012 
[25] Surya Cheemalapati, Michael Gubanov, Michael Del Vale and Anna Pyayt, "A Real-Time Classification Algorithm for Emotion Detection using Portable EEG", Proceedings of IEEE International Conference on Information Reuse and Integration, pp. 720-723, 2013.

[26] Y. Gu, S.L. Tan, K.J. Wong, M.H. R. Ho and L. Qu, "Using GA-based Feature Selection for Emotion Recognition from Physiological Signals", Proceedings of IEEE International Symposium on Intelligent Signal Processing and Communications Systems, pages 1-4, 2008.

[27] Aayush Bhardwaj, Ankit Gupta, Pallav Jain, Asha Rani and Jyoti Yadav, "Classification of Human Emotions from EEG signals using SVM and LDA Classifiers", Proceedings of IEEE International Conference on Signal Processing and Integrated Networks, pp. 180-185, 2015.

[28] Khadidja Gouizi, Fethi Bereksi Reguig and Choubeila Maaoui, "Analysis Physiological Signals for Emotion Recognition”, Proceedings of IEEE International Workshop on Systems, Signal Processing and their Applications, pp.147-150, 2011.

[29] T. Baby Shalini and L. Vanitha, "Emotion Detection in Human Beings using ECG Signals", International Journal of Engineering Trends and Technology, Vol. 4, No. 3, pp. 12-18, 2013.

[30] Zhengjie Zhou, Huiping Jiang and Xiaoyuan Song, "EEGbased Emotion Recognition using Wavelet Features", Proceedings of IEEE International Conference on Software Engineering and Service Science, pp. 585-588, 2014.

[31] Kazuhiko Takahashi, "Remarks on SVM-based Emotion Recognition from Multi-Modal Bio-Potential Signals", Proceedings of IEEE International Workshop on Robot and Human Interactive Communication, pp. 95-100, 2004.

[32] Reza Khosrowabadi, Chai Quek, Kai Keng Ang and Abdul Wahab, "ERNN: A Biologically Inspired Feed Forward Neural Network to Discriminate Emotion from EEG Signal", IEEE Transactions on Neural Networks and Learning Systems, Vol. 25, No. 3, pp. 223-233, 2014.
[33] Chendi Wang and Feng Wang, "An Emotional Analysis Method based on Heart Rate Variability", Proceedings of IEEE International Conference on Biomedical and Health Informatics, pp. 1-7, 2012.

[34] Chang-wei Ma and Guang-yuan Liu, "Feature Extraction, Feature Selection and Classification from Electrocardiography to Emotions", Proceedings of IEEE International Conference on Computational Intelligence and Natural Computing, Vol. 1, pp. 190-193, 2009.

[35] Guangying Yang and Shanxiao Yang, "Study of Emotion Recognition based on Surface Electromyography and Improved Least Squares Support Vector Machine", Journal of Computers, Vol. 6, No. 8, pp. 112-129, 2011.

[36] Ruo-Nan Duan, Jia-Yi Zhu and Bao-Liang Lu, "Differential Entropy Feature for EEG-based Emotion Classification", Proceedings of IEEE International Conference on Neural Engineering, pp. 81-84, 2013.

[37] Xizhi Zhu, "Emotion Recognition of EMG based on BP Neural Network", Proceedings of $2^{\text {nd }}$ International Symposium on Networking and Network Security, pp. 1-4, 2010.

[38] Somchanok Tivatansakul and Michiko Ohkura, "Emotion Recognition using ECG Signals with Local Pattern Description Methods", International Journal of Affective Engineering, Vol. 15, No. 2, pp. 51-61, 2016.

[39] Sabaa Ahmed Yahya Al-Galal, Imad Fakhri Alshaikhli and Abdul Wahab Abdul Rahman, "Automatic Emotion Recognition based on EEG and ECG Signals While Listening to Quranic Recitation Compared with Listening to Music", Proceedings of $6^{\text {th }}$ International Conference on Information and Communication Technology for The Muslim World, pp. 1-4, 2016.

[40] Mehmet Ali Sarkaya and Gokhanince, "Emotion Recognition from EEG Signals through One Electrode Device", Proceedings of IEEE 25 th Signal Processing and Communications Applications Conference, pp. 1-6, 2017. 